\title{
RNA Interference Reveals the Coregulatory Effects of Cylindromatosis on Apoptosis and Necroptosis of Photoreceptor Cells in Experimental Retinal Detachment
}

\author{
Kai Dong, ${ }^{* \dagger}$ Linfeng Han, ${ }^{*}$ Jingwen Liu, ${ }^{*}$ Fenghua Wang, ${ }^{\dagger}$ and Xiaodong Sun ${ }^{\dagger \dagger}$
}

\begin{abstract}
From the Department of Ophthalmology, * Anhui Provincial Hospital, Anhui Medical University, Hefei; the Department of Ophthalmology, ${ }^{\dagger}$ Shanghai First People’s Hospital, School of Medicine, Shanghai Jiaotong University, Shanghai; and the Laboratory of Molecular Biology, ${ }^{\ddagger}$ Shanghai Key Laboratory of Fundus Disease, Shanghai, China
\end{abstract}

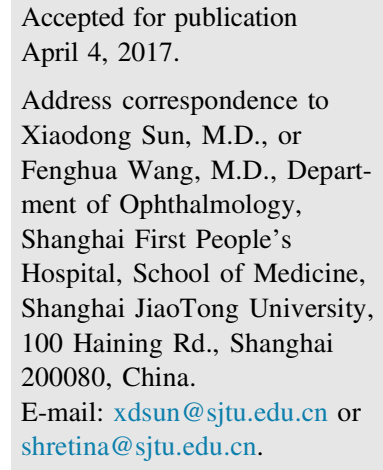

\begin{abstract}
Inhibiting only cell apoptosis or necroptosis in photoreceptor cells does not protect them against death after traumatic retinal detachment. This study was designed to evaluate the coregulatory effects of the deubiquitinating enzyme cylindromatosis on the apoptosis and necroptosis of photoreceptor cells in experimental retinal detachment. Lentivirus Cyld shRNA was generated and used to suppress cylindromatosis expression in Sprague-Dawley rats. Three weeks after injection of lentivirus Cyld shRNA, retinal detachment surgery was performed. Transmission electron microscopy, propidium iodide staining, terminal deoxynucleotidyl transferase-mediated dUTP nick-end labeling assay, electroretinography, and determination of ubiquitination and phosphorylation of receptor-interacting protein 1 were performed to detect the apoptosis and necroptosis of photoreceptor cells. Knockdown of cylindromatosis expression led to inhibition of caspase 8 activity, a decrease in the number of apoptotic photoreceptor cells, and an increase in the ubiquitination level of receptor-interacting protein 1 . In addition, the number of necroptotic cells decreased and the phosphorylation level of receptorinteracting protein 1 decreased dramatically; significant protective effects of RNA interferencemediated suppression of cylindromatosis expression on electroretinogram wave were observed. Cylindromatosis coregulates the apoptosis and necroptosis of photoreceptor cells by regulating the ubiquitination of receptor-interacting protein 1 after retinal detachment. (Am J Pathol 2017, 187: 1763-1771; http://dx.doi.org/10.1016/j.ajpath.2017.04.015)
\end{abstract}

Retinal detachment (RD) is a common ophthalmic disease that is caused mainly by photoreceptor cell death and may lead to irreversible defects in visual function. The mechanisms of photoreceptor cell death depend mainly on the death receptors that induce apoptosis and necroptosis.

Previous studies have reported that some mediators can induce cell death in an experimental RD model. For example, an apoptosis-inducing factor is translocated from the mitochondrial intermembrane space to the nucleus to induce photoreceptor cell apoptosis. ${ }^{2}$ Caspase activation is associated with photoreceptor cell apoptosis in experimental RD. ${ }^{3}$ Our previous study showed that z-VAD-FMK [benzyloxycarbonylVal-Ala-DL-Asp(O-methyl)-fluoromethylketone], a pan caspase inhibitor, not only inhibited photoreceptor cell apoptosis, but also induced necroptosis by down-regulating receptor interacting protein-1 (RIP1) phosphorylation in photoreceptor cells after experimental $\mathrm{RD},{ }^{4}$ whereas necrostatin-1 specifically inhibited necroptosis, but did not affect apoptosis. ${ }^{5}$ Trichonas and Donahoe ${ }^{6}$ indicated that

\footnotetext{
Supported by The National Science Fund for Distinguished Young Scholars of China grant 81425006, Natural Science Foundation of Anhui Province grant 1408085QH159, National Natural Science Foundation of China grants 81400407 and 30973259, and Shanghai Scholar Leadership Foundation grant XBR2013081.
}

Disclosures: None declared. 
in addition to apoptosis, simultaneous inhibition of necrosis is essential for effective protection of photoreceptor cells after photoreceptor detachment. Therefore, novel targets that simultaneously inhibit apoptosis and necroptosis are required urgently for therapeutic intervention to protect against photoreceptor cell apoptosis and necroptosis in the retina after traumatic RD.

Previous studies have shown that apoptosis and necroptosis both occur via RIP1 in death-receptor signaling pathways. $^{7-9}$ Protein deubiquitination is important for signal transduction during RIP1-mediated apoptosis and necroptosis. Cylindromatosis (CYLD) is a major deubiquitinating enzyme in tumor necrosis factor-receptormediated cell death. ${ }^{8,10-13}$ However, whether CYLD plays a role in controlling the apoptosis and necroptosis of photoreceptor cells by affecting RIP1 ubiquitination remains unclear. The goal of this study was to investigate the impact of down-regulation of CYLD expression on the apoptosis and necroptosis of retina cells by using RNA interference (RNAi). The common targets and regulatory mechanisms involved in both apoptosis and necroptosis of photoreceptor cells in RD models were evaluated further, which may be helpful for inhibiting different types of cell death using a single inhibitor.

\section{Materials and Methods}

\section{Animals and Ethical Approval}

All animal experiments were approved by the Animal Research Committee at Shanghai Jiaotong University and conducted with strict adherence to the Association for Research in Vision and Ophthalmology Statement for the Use of Animals in Ophthalmic and Vision Research.

Healthy male Sprague-Dawley rats (weight, 260 to $280 \mathrm{~g}$ ) were provided by Shanghai First People's Hospital of Shanghai Jiaotong University.

\section{CYLD Expression after RD}

To examine CYLD protein expression after RD, normal male Sprague-Dawley rats were assigned to six groups (with six rats in each group): normal control, and 1, 3, 5, 7, and 14 days after RD. RD was induced only in the right eye of each rat as described in our previous study, ${ }^{4}$ with the contralateral left eyes serving as controls. Specimens were obtained from each group at the end of each observation period. Western blot analysis was performed as previously described, ${ }^{14}$ using $\beta$-actin as an internal standard.

\section{Generation of GFP-Cyld-shRNA Lentivirus}

Lentiviral transfer vector driven by the Rous sarcoma virus promoter, U6, and cytomegalomavirus promoter in sterile distilled water was conducted. The packaging plasmids were pRsv-REV and pMDlg-pRRE, and the envelope plasmid was pMD2G. The green fluorescent protein (GFP)-lentiviral vectors encoding shRNAs against rat $C y l d$ were synthesized by Telebio Biomedical Co., Ltd. (Shanghai, China). A lentivirus expression system was used to prepare vector particles. Three lentivirus shRNAs targeting different sites of $C y l d$ were designed according to the nucleotide sequence of Cyld (https://www.ncbi.nlm.nih.gov/nuccore/; accession number NM_001017380.1) as follows: Cyld-rat-801 (Cyld1): 5'-GGAGCTTATAGAAGATGATGA-3'; Cyldrat-1720(Cyld2): 5'-GGTTGTACGGATGGAACTTTC-3'; Cyld-rat-2100(Cyld3): 5'-GCTACTGAGGACAGAGATAGT-3', and a negative control lentivirus shRNA: 5' TTCTCCGAACGTGTCACGT-3'. choroid-RPE-2240 cells were transduced with these four lentivirus shRNAs and Western blot analysis was performed to test the interference effect. Transfection efficacies of all samples were greater than 95\%. The lentivirus Cyld shRNA-2 (LV-sh2) showed the best silencing efficacy and was chosen for subsequent in vivo experiments.

\section{Subretinal Injection with shRNA Lentiviruses}

According to different types of interventions, male SpragueDawley rats were divided into an untreated (uninfected by lentivirus), balanced salt solution, infected with CyldshRNA lentiviruses (sh-Cyld), and infected with negative control lentivirus shRNA (sh-neg) groups. The right eyes were used as experimental eyes and the contralateral left eyes were used as control eyes. Three weeks after lentivirus infection, the specimens were harvested.

The experimental rats were anesthetized with chloral hydrate $(10 \%, 400 \mathrm{mg} / \mathrm{kg}$ intraperitoneally). Preoperative mydriasis was accomplished with $0.5 \%$ tropicamide and 0.5\% epinephrine (Santen Pharmaceutical Co., Ltd., Suzhou, China). Preoperative disinfection of the conjunctival sac was performed with levofloxacin eyedrops (Santen Pharmaceutical Co., Ltd.). Subretinal injection was conducted as previously described ${ }^{15-18}(5-\mu \mathrm{L}$ vector suspension containing $10^{8}$ transducing units $/ \mathrm{mL}$ ). Briefly, the rat cornea was fitted with a corneal contact lens and the sclera and choroid were punctured. A subretinal injection of $3 \times 10^{8} \mathrm{IU} / \mathrm{mL}$ lentivirus $(3 \mu \mathrm{L})$ was introduced slowly with a 30 -gauge needle. ${ }^{15-18}$ A slight bulge in the retina was observed under a microscope and ocular inflammation was prevented by administration of levofloxacin eyedrops three times per day. ${ }^{19}$

\section{Hematoxylin and Eosin Staining and Fluorescence Detection in Rats in Batch 1}

Three weeks after injection, the rats in batch 1 were sacrificed after being anesthetized with $10 \%$ chloral hydrate and their eyeballs were removed rapidly. The retinal vasculature was stained with hematoxylin and eosin and then examined and photographed. ${ }^{20}$ Optical imaging of GFP fluorescence in each sample was conducted using a confocal laser scanning microscope (LSM 510; Zeiss, Jena, Germany). 


\section{RD Surgery in Groups of Rats in Batch 2}

Batch 2 of rats was divided into the following four groups: attached group (uninfected with lentivirus and received no $\mathrm{RD}$ surgery), untreated group (uninfected with lentivirus and received RD surgery), sh-Cyld group (infected with LV-sh2 and received RD surgery), and sh-neg group (infected with LV-sh-neg and received RD surgery). Experiments were performed in the left eyes and the contralateral right eyes were considered to be controls.

The RD model was prepared as described previously ${ }^{4}$ and generated 3 weeks after lentivirus injection. Briefly, the rats were anesthetized and their pupils were dilated as described earlier. RDs were enlarged via subretinal injection of $1 \%$ sodium hyaluronate (Bausch \& Lomb Freda, Beijing, China) $(50 \mu \mathrm{L}$ each) into the subretinal space using a 30 gauge needle. ${ }^{2}$ In addition, specimens were obtained and examined 3 days after RD surgery. Immunohistochemical analysis of CYLD in the retina was performed according to the instruction manual (Gene Tech Co., Ltd., Shanghai, China) based on previous studies. ${ }^{2,21,22}$ Image-Pro Plus software version 6.0 (Media Cybernetics, Inc., Rockville, MD) was used to measure integrated optical density (IOD).

\section{Morphologic Evaluation of Rats in Batch 2}

RD-induced apoptotic morphologic alterations in the photoreceptors were observed by transmission electron microscopy. Briefly, the eyes were immersed fully in a cold $\left(4^{\circ} \mathrm{C}\right) 4 \%$ glutaraldehyde $(0.1 \mathrm{~mol} / \mathrm{L}$ phosphate buffer, $\mathrm{pH}$ 7.4) solution and incubated for 24 hours. The dissected retinas were fixed afterward with $1 \%$ osmium tetroxide in $0.1 \mathrm{~mol} / \mathrm{L}$ sodium phosphate buffer ( $\mathrm{pH} 7.2)$, followed by dehydration in ethanol and water, and embedded in Eponate (Ted Pella, Inc., Redding, CA). The retina was observed and photographed using a JEM1200EX electron microscope (JEM, Tokyo, Japan). Typically, the apoptotic morphology of photoreceptors appears as cellular shrinkage and nuclear condensation, whereas necroptotic cells show swelling and rapid plasma membrane expansion. ${ }^{6,23}$

\section{Membrane Integrity Evaluation}

In addition, propidium iodide (PI; Sigma, St. Louis, MO) staining was used to evaluate the membrane integrity of photoreceptor cells as previously described ${ }^{6,24,25}$ in four groups of batch 2. Briefly, PI was diluted twofold with double-distilled $\mathrm{H}_{2} \mathrm{O}$. Five microliters of PI $(50 \mu \mathrm{g} / \mathrm{mL})$ was injected into the subretinal space 2 hours before sacrifice. Next, the eyes were enucleated and $10-\mu \mathrm{m}-$ thick cryosections were cut and air-dried. The PI-labeled specimens were fixed with $100 \%$ ethanol for 10 minutes at room temperature. DAPI was used to counterstain the nucleus. Red fluorescence of PI-positive cells was observed by a confocal laser scanning microscope (LSM 510; Zeiss) and counted using Image-Pro Plus software version 6.0 (Media Cybernetics, Inc.). Moreover, a terminal deoxynucleotidyl transferase-mediated dUTP nick-end labeling (TUNEL) assay was conducted to investigate DNA damage (apoptosis) in photoreceptor cells. TUNEL-positive cells were observed and counted as described earlier.

\section{Rats of Batch 2 Were Determined by ERG}

In addition, 7 days before and 7 days after RD surgery, electroretinography (ERG) signals were assessed for rats in each group (EP-1000; Tomey, Erlangen, Germany) as described previously. ${ }^{26}$ The a-wave was measured from the baseline to the point of maximum initial negative response, whereas the b-wave was from the peak of the a-wave to the positive peak.

\section{Immunoprecipitation and Western Blot Analysis}

Samples were run on $8 \%$ SDS-PAGE. After electrophoretic separation, the proteins were electrotransferred onto nitrocellulose membranes (Whatman, Maidstone, UK). The nitrocellulose membrane was blocked by incubation with $5 \%$ bovine serum albumin in Tris-buffered saline and $0.02 \%$ Tween-20 (pH 7.4) for 2 hours at room temperature. The blotting membranes were reacted with RIP1 (1:1000, cata$\log$ number 3493; Cell Signaling Technology, Danvers, MA), phosphoserine (1:100, catalog number ALX-804-165C100; Enzo Life Sciences, Farmingdale, NY), caspase-8 (1:1000, catalog number 49-788; Prosci, Inc., Poway, CA), CYLD (1 to $2 \mathrm{mg} / \mathrm{mL}$, catalog number SAB4200060; Sigma), and $\beta$-actin (Sigma-Aldrich Chemie $\mathrm{GmbH}$, Munich, Germany) antibodies, respectively. Membranes then were washed three times and incubated with horseradish-peroxidase-labeled secondary antibody (diluted 1:5000 in Tris-buffered saline and 0.02\% Tween-20; Santa Cruz Biotechnology, Santa Cruz, CA) for 2 hours at room temperature. Bands were visualized using electrochemiluminescence (Amersham Pharmacia Biotech, Amersham, UK) according to the manufacturer's instructions and were exposed to X-ray film. Signal density was quantified using Bandscan software version 4.3 (Glyko, Inc., Novato, CA).

\section{Ubiquitination and Phosphorylation of RIP1 in Rats in} Batch 2

RIP1 ubiquitination was determined by coimmunoprecipitation experiments. ${ }^{27,28}$ The grayscale values of each protein band were semiquantified by Bandscan43 (Glyko, Inc.). RIP1 phosphorylation was detected by immunoprecipitation in conjunction with Western blot analysis using a phosphospecific RIP1 kinase antibody. ${ }^{29}$

\section{Statistical Analysis}

Data were presented as the means $\pm \mathrm{SD}$ and analyzed using SPSS 17.0 software (SPSS, Inc., Chicago, IL). Cell counts 
obtained by electron microscope, TUNEL, and PI analysis, as well as results of Western blot assay and others, were compared across groups using multivariate analysis of variance. $P<0.05$ was considered statistically significant.

\section{Results}

\section{CYLD Expression after Experimental RD}

The Western blot analysis showed that CYLD protein levels increased after RD surgery (Figure 1, A and C). The highest expression level was observed on day $3(P<0.01)$, and then the protein level gradually decreased and returned to baseline level on day 14. The interference effect of three lentivirus shRNAs is shown in Figure 1, B and D. LV-sh2 produced the strongest interference effects (efficiency, approximately $80 \%$ ), whereas that of LV-sh1 was the weakest.

\section{Morphologic Changes after Subretinal Injection with shRNA Lentiviruses}

Localized RD was observed after LV-sh2 injection (Figure 1E) compared with that in the control eye (Figure $1 \mathrm{~F}$ ) and lasted for 7 to 10 days. The retina was fully reattached within 3 weeks. Three weeks later, hematoxylin and eosin staining showed no significant difference in the retinal vasculature among the untreated, balanced salt solution, sh-Cyld, and sh-neg groups (Figure 1, G-J).

\section{GFP Fluorescence-Based Validation of shRNA Lentivirus Infection}

Three weeks after lentivirus injection, GFP fluorescence was used to observe the retinal cells infected by recombinant lentivirus vectors carrying the GFP gene. Figure 2, A-C, shows the GFP fluorescence in retinal tissues infected with LV-sh2 for 3 weeks, particularly in the outer nuclear layer.

\section{CYLD Expression Is Inhibited by LV-sh2 in the RD Models}

Three days after RD surgery, the CYLD protein level in the sh-Cyld group was significantly lower than that in the untreated and sh-neg groups (Figure 2, D and E). In addition, immunohistochemical staining for CYLD indicated that CYLD expression increased in the untreated group (IOD, $669.50 \pm 124.65$ ) and the sh-neg group (IOD, $710.00 \pm 44.32$ ) compared with that in the attached group (IOD, $236.50 \pm 61.20$ ), whereas the protein level decreased

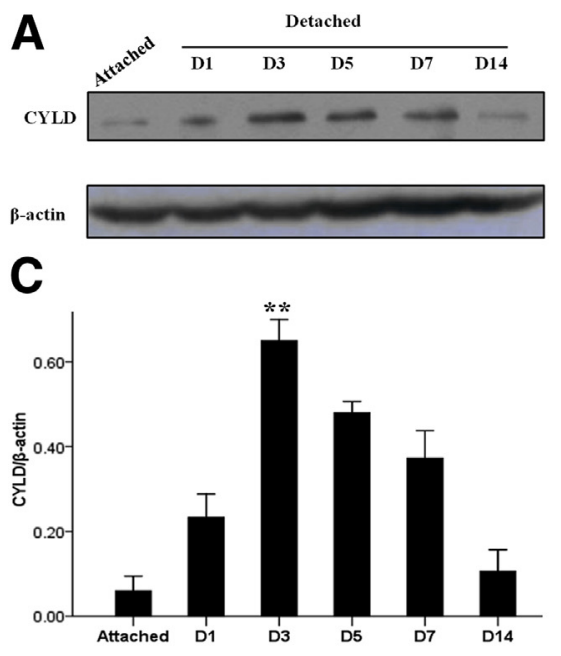

E
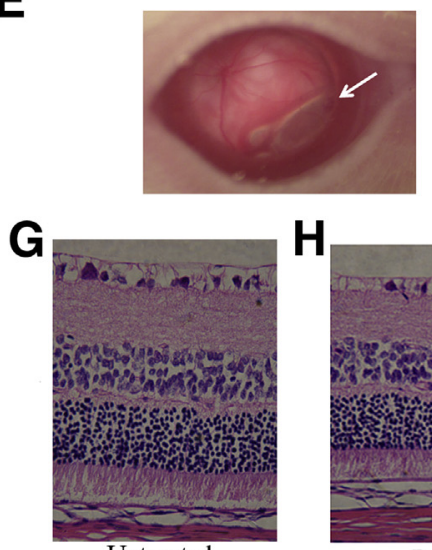

Untreated

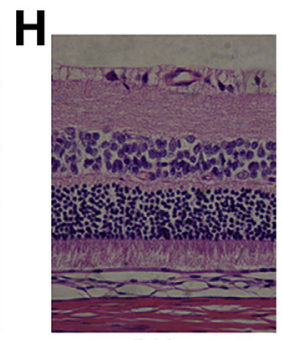

BSS
B

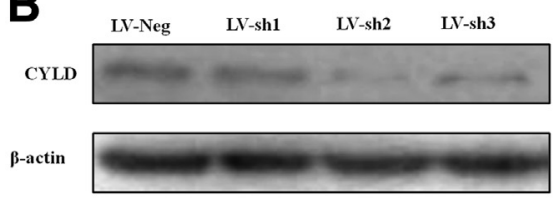

D。

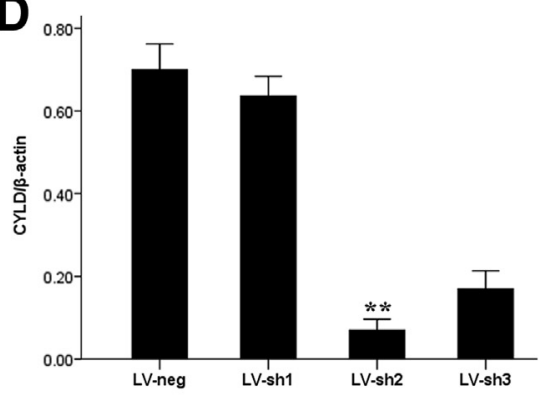

$\mathbf{F}$
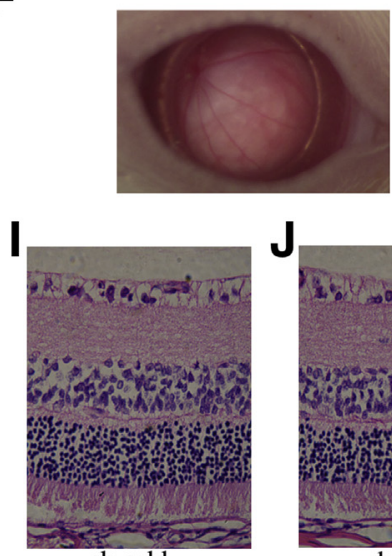

sh-cyld

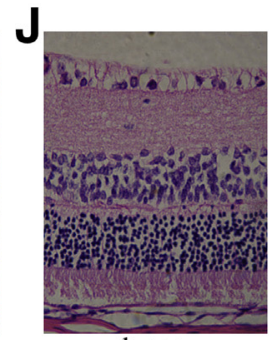

sh-neg
Figure 1 Cylindromatosis (CYLD) expression and morphologic changes in groups. Expression of CYLD protein after experimental retinal detachment by Western blot analysis (A) and the integrated optical density value $(\mathbf{C})$. The measurement of lentiviral shRNA interference efficiency in choroid-RPE 2240 cells by Western blot analysis (B) and the integrated optical density value (D). Fundus changes in experimental (arrow) (E) compared with control (F) eyes after lentiviral LVsh2 injection (magnification 8). Retinal vasculature changes in each group: untreated (G), balanced salt solution (BSS) treated (H), sh-cyld $(\mathbf{I})$, and sh-neg $(\mathbf{J})$ at 3 weeks after subretinal injection of lentiviral vectors. ${ }^{* *} P<0.01$ versus all other groups (analysis of variance). Original magnification: $\times 8(\mathbf{E}$ and $\mathbf{F}) ; \times 200(\mathbf{G}-\mathbf{J})$. D, day. 


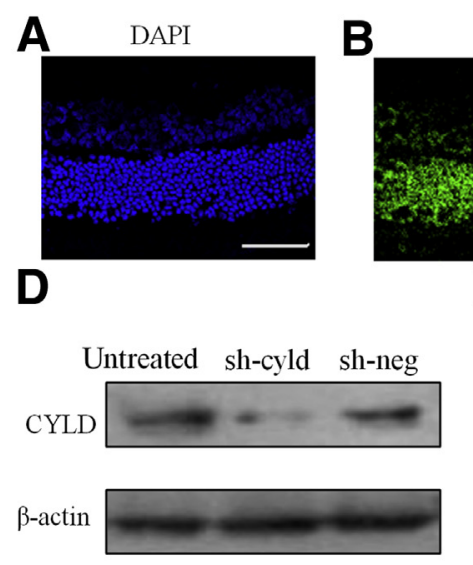

$\mathbf{F}$

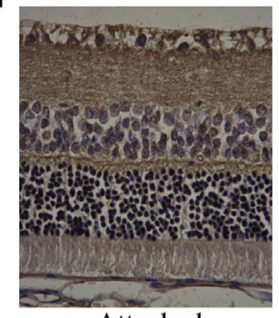

Attached

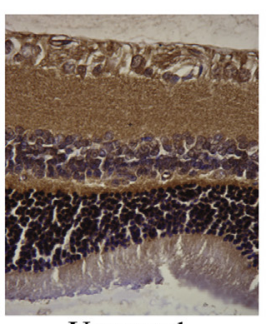

Untreated
GFP

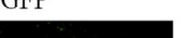

E
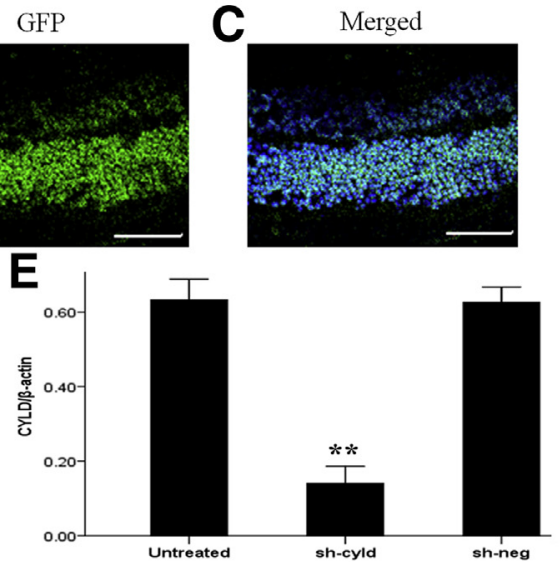

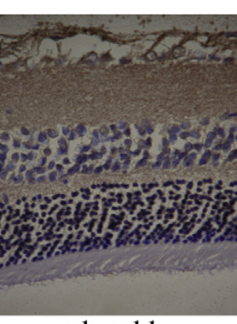

sh-cyld

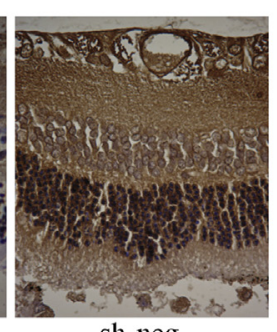

sh-neg
Figure 2 Transfection efficiency and interference efficiency of lentiviral-shRNA2. A: Inner and outer nuclear layers of the retina with DAPI staining. $\mathbf{B}$ and $\mathbf{C}$ : Expression of green fluorescent protein (GFP) protein 3 weeks after lentiviral vector subretinal injection. Expression of cylindromatosis (CYLD) protein 3 days after experimental retinal detachment by Western blot analysis (D) and the integrated optical density value (E). Expression and location of CYLD protein 3 days after experimental retinal detachment by immunohistochemistry $(\mathbf{F})$ and the integrated optical density (IOD) value (G). ${ }^{* *} P<0.01$ versus untreated and sh-neg group (analysis of variance). Scale bars $=50 \mu \mathrm{m}$ $(A-C)$. Original magnification, $\times 200(\mathbf{F})$. in the sh-Cyld group (IOD, $215.33 \pm 56.69$ ). The most obvious differences were observed in the outer nuclear layer (all $P<0.01$ ) (Figure 2, F and $\mathrm{G}$ ).

\section{Effects of Decreased CYLD Expression on Photoreceptor Cell Apoptosis in RD Models}

To identify the effects of suppressed expression of CYLD on photoreceptor cells, apoptotic changes were determined by the TUNEL assay. The number of TUNEL-positive photoreceptor cells in the sh-Cyld group (618.80 \pm 109.53 / $\mathrm{mm}^{2}$ ) was lower than those in the untreated group $\left(1409.69 \pm 282.45 / \mathrm{mm}^{2}\right)$ and the sh-neg group $\left(1390.75 \pm 208.49 / \mathrm{mm}^{2} ; P<0.01\right)$ (Figure 3, A and B).

Because RIP1 ubiquitination is required for the activation of caspase 8 in the death receptor-mediated apoptosis pathway, the effects of $C Y L D$ knockdown on RIP1 ubiquitination and caspase 8 activation were determined. Immuno-coprecipitation assays showed that the ubiquitination level of RIP1 in the sh-Cyld group $(0.85 \pm 0.04)$ was relatively high, whereas the level in the untreated group $(0.23 \pm 0.04)$ and the sh-neg group $(0.24 \pm 0.03)$ decreased compared with the values for the sh-Cyld group or attached group (all $P<0.01$ ) (Figure 3, C and D).

Western blot results showed that activated caspase 8 markedly decreased in the sh-Cyld group (0.14 \pm 0.04$)$, compared with that in the sh-neg group $(0.71 \pm 0.05$; $P<0.01)$. There were no significant differences in the activated caspase 8 level between the sh-neg group and the untreated group $(P>0.05)$ (Figure 3, E and F).
Effects of Decreased CYLD Expression on Photoreceptor Cell Necroptosis in RD Models

Whether Cyld RNAi affects the necroptosis of photoreceptor cells also was examined. PI staining showed that PI-positive photoreceptors in the sh-Cyld group $\left(439.25 \pm 67.09 / \mathrm{mm}^{2}\right)$ were significantly lower than those in the untreated group $\left(809.75 \pm 91.26 / \mathrm{mm}^{2}\right)$ and the sh-neg group $\left(808.01 \pm 69.97 / \mathrm{mm}^{2} ; P<0.01\right)$ (Figure 4, A and B).

Morphologic changes in photoreceptor cells also were observed. Transmission electron microscopy analysis showed similar results as those of PI staining: necrosis of photoreceptor cells significantly decreased in the sh-Cyld group compared with that in the untreated group and the sh-neg group $(P<0.01)$ (Figure $4, \mathrm{C}$ and $\mathrm{D}$ ).

RIP1 phosphorylation regulates programed necroptosis. Thus, RIP1 phosphorylation was analyzed further in an immuno-coprecipitation assay. The level of phosphorylated RIP1 in the sh-Cyld group was significantly lower than that in the attached group, whereas no obvious inhibitory influence on RIP1 phosphorylation was observed in the untreated group or the sh-neg group $(P<0.01)$ (Figure 5, A and B). In addition, the effects of CYLD knockdown on NF$\kappa \mathrm{B}$ expression were analyzed. The results showed that NF$\kappa \mathrm{B}$ p65 expression increased after Cyld knockdown (Figure 5, C and D). To determine whether the inhibition effects of decreased CYLD expression on photoreceptor cell necroptosis could protect retinal function, ERG analysis, an electrophysiological measure of bipolar cell function, was conducted. Seven days after experimental RD, the a-wave of the sh-Cyld group recovered to $65.50 \%$ of baseline value, 


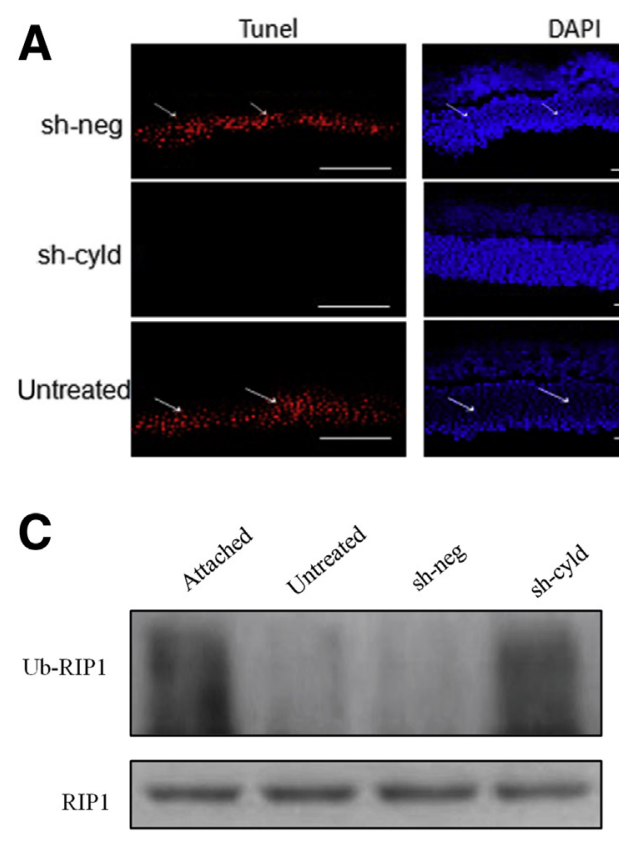

E

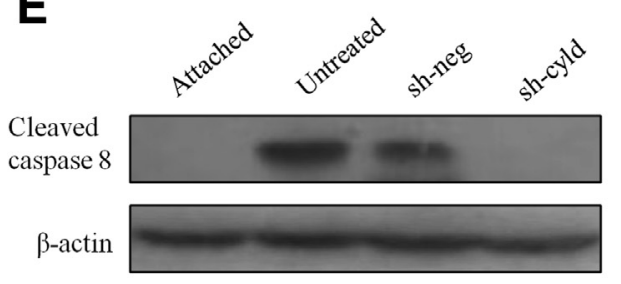

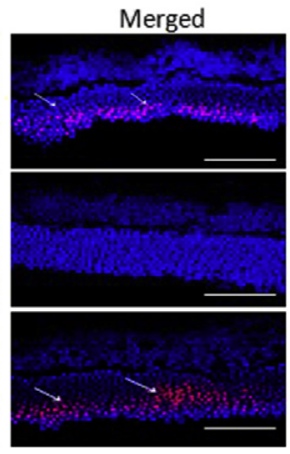

B

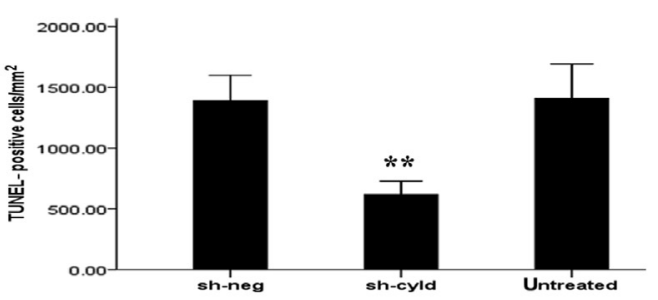

D
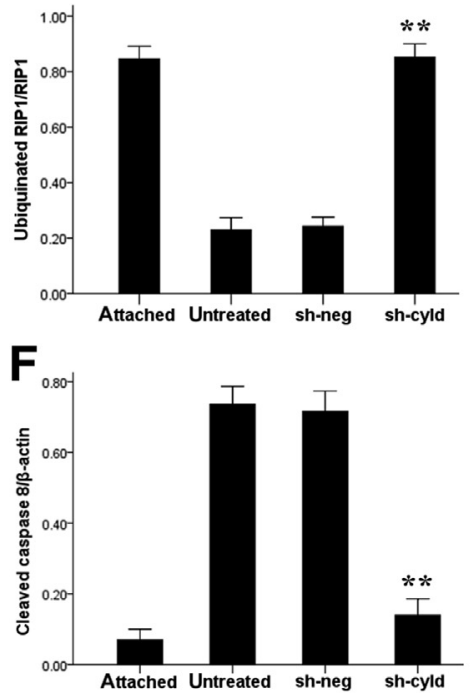

Figure 3 Effects of decreased cylindromatosis (CYLD) expression on photoreceptor cell apoptosis after experimental retinal detachment. Terminal deoxynucleotidyl transferase-mediated dUTP nickend labeling (TUNEL) analysis 3 days after experimental retinal detachment $(\mathbf{A})$ and the number of TUNEL-positive cells (B). Arrows indicate the red apoptotic cells. Receptor interacting protein-1 (RIP1) ubiquitination among groups 3 days after experimental retinal detachment $(\mathbf{C})$ and the integrated optical density value (D). Caspase 8 activation 3 days after experimental retinal detachment by Western blot analysis (E) and the integrated optical density value $(\mathbf{F})$. ${ }^{* *} P<0.01$ versus both the untreated and the sh-neg group (analysis of variance). Scale bars $=50 \mu \mathrm{m}(\mathbf{A})$. which was higher than that in the untreated group $(31.20 \% \pm 0.07 \%)$ and the sh-neg group $(30.25 \% \pm 0.09 \%$; $P<0.01$ ) (Figure 5, E and F). A similar protective effect on the ERG b-wave was observed (Figure 5, E and G).

\section{Discussion}

In our study, the CYLD protein level after experimental RD in rats increased continuously and reached a peak concentration on day 3. This is consistent with the high cell death rate. In addition, RIP1 deubiquitination was associated with an up-regulated expression of CYLD and accompanied cell death. The results suggest that CYLD is important for photoreceptor cell death. Recovery of visual function in patients after RD is not ideal, mainly because of the irreversible death of photoreceptor cells. Apoptosis and necrosis are two major cell death modalities, and inhibiting only one of these modalities does not protect photoreceptor cells effectively against death. Therefore, identifying a common target for apoptosis and necrosis of photoreceptor cells may be helpful in suppressing cell death. In this study, CYLD knockdown inhibited not only apoptosis, but also necrosis of photoreceptor cells. This may provide a new direction for effective recovery of the visual function of RD patients.

The ubiquitination status of RIP1 in death-receptor signaling determines the initiation of a death cascade or survival signal. ${ }^{30}$ RIP1 ubiquitination facilitates cancer cell survival, whereas deubiquitination of RIP1 accelerates cell apoptosis. ${ }^{30}$ CYLD is the deubiquitinating enzyme related most closely to RIP1. ${ }^{8,10-13}$ An up-regulated expression of CYLD induces the deubiquitination of RIP, and the deubiquitinated RIP1 and the adaptor protein FADD are recruited to complex IIa or complex IIb with RIP3 to regulate cell death via the intrinsic apoptotic/necroptotic pathway. Our results showed that z-VAD-FMK can inhibit apoptosis by inhibiting the activation of caspase 8 , and Necrostatin-1 can inhibit necroptosis by inhibiting the phosphorylation of RIP1. However, z-VAD-FMK and Necrostatin-1 cannot suppress cell death (Figure 6). The complex IIa activates caspase 8 to induce cell apoptosis. ${ }^{11}$ Bignell et $\mathrm{al}^{31}$ found that decreased expression of CYLD is conducive for cell survival via an RIP1-dependent mechanism. The binding of RIP1 to FADD is thought to be responsible for the recruitment and activation of caspase 


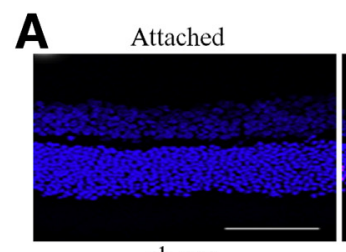

sh-neg
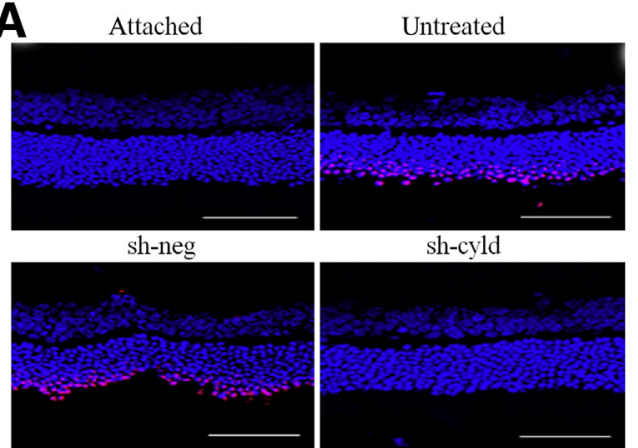

sh-cyld
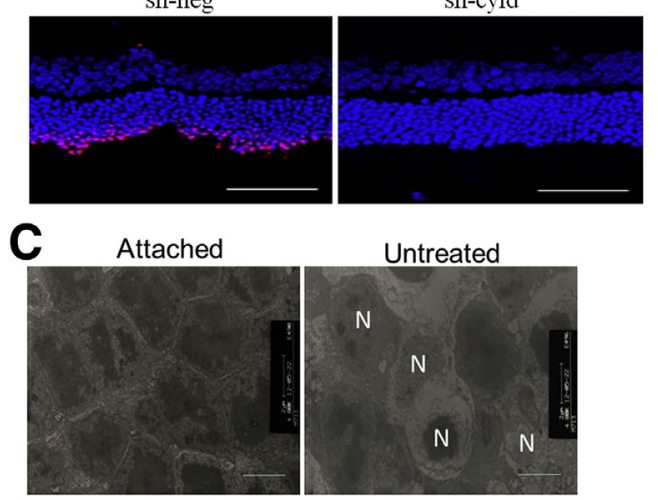

sh-neg

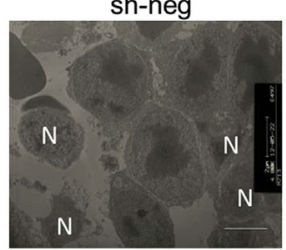

Untreated

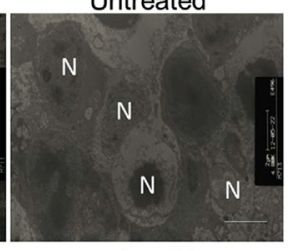

sh-cyld

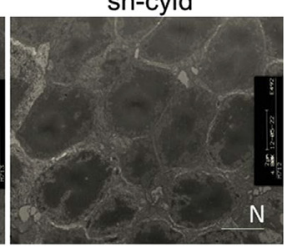

B

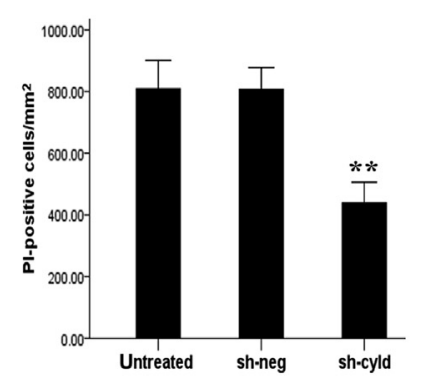

D

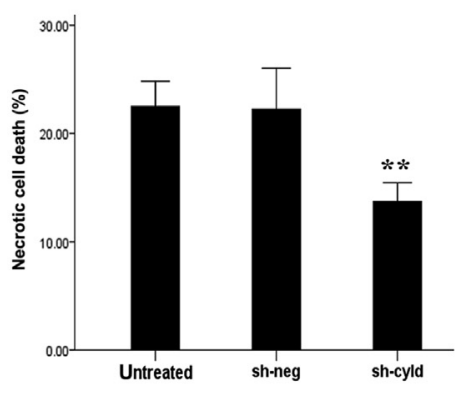

Figure 4 Effects of decreased cylindromatosis (CYLD) expression on photoreceptor cell necroptosis after experimental retinal detachment. Propidium iodide (PI) staining 3 days after experimental retinal detachment $(\mathbf{A})$ and the integrated optical density value (B). Transmission electron microscopy analysis among groups 3 days after experimental retinal detachment $(\mathbf{C})$, and the integrated optical density value (D). ${ }^{* *} P<0.01$ versus untreated and sh-neg group (analysis of variance). Scale bars: $50 \mu \mathrm{m}$ (A); $2 \mu \mathrm{m}$ (C). N, necroptotic morphology of photoreceptor cells.
8 , and results in the signaling cascade that leads to apoptotic cell death. $^{32,33}$ Wang et al $^{11}$ suggested that CYLD suppression by RNAi could suppress the apoptosis induced by tumor necrosis factor $\alpha$ and inhibit activation of caspase 8 , and suggested that ubiquitinated RIP1, induced by CYLD, could bind to FADD to form complex IIa, and activate caspase 8, finally inducing the conduction of apoptosis signals. Thus, we predicted that expression of CYLD would be increased after RD, and RIP1 would be deubiquitinated to form complex IIa with FADD to regulate the apoptotic signal and result in photoreceptor cell death.

In this study, after shRNA lentivirus transfection, CYLD expression in retinal cells was inhibited and the ubiquitination level of RIP1 increased. The TUNEL assay showed that the number of apoptotic photoreceptor cells decreased after knockdown of CYLD expression. Moreover, downregulated CYLD expression also significantly suppressed caspase 8 activation. This is the first study to show the inhibition effects of $C Y L D$ knockdown on photoreceptor cell apoptosis after RD by reducing the activation of caspase 8 . In addition, RIP1 ubiquitination and phosphorylation are important for the necroptotic signaling pathway executed by complex IIb. $^{34,35}$ Thus, the increased ubiquitination level of RIP1 also would contribute to the occurrence of photoreceptor cell necroptosis after RD. Hitomi et $\mathrm{al}^{8}$ found that decreased expression of CYLD not only inhibited cell apoptosis, but also regulated Z-VAD-FMK-induced necroptosis. Thus, we also suggested that an increased level of
CYLD after RD would deubiquitinate RIP1, which forms complex IIb with RIP3 to induce necroptosis of photoreceptor cells. The study focused on the regulatory effect of CYLD expression on photoreceptor cells and showed that after RNAi-mediated suppression of CYLD expression, the number of PI-positive cells and necrosis-like forms of cell death decreased. In addition, because phosphorylated RIP1 is the protein marker for necroptosis, the effects of suppressed CYLD expression on RIP1 phosphorylation were analyzed. We found that the phosphorylation level of RIP1 decreased dramatically. Moreover, significant protective effects of knockdown of CYLD expression on ERG waves were observed. Therefore, decreased CYLD expression in RD rats may inhibit necroptotic cell death by increasing ubiquitination and inhibiting phosphorylation of RIP1. In addition, Trompouki et $\mathrm{al}^{12}$ showed that CYLD, a ubiquitination enzyme, negatively regulates the activity of NF$\kappa \mathrm{B}$, inhibits the expression of CYLD protein, and activates the NF- $\kappa \mathrm{B}$ pathway. Thus, the expression of NF- $\kappa \mathrm{B}$ was investigated further and the results suggested that the expression of NF- $\kappa \mathrm{B}$ p65 increased after Cyld knockdown. This indicates that the protective effects of CYLD on photoreceptor cells are mediated by $\mathrm{NF}-\kappa \mathrm{B}$ activation.

In conclusion, by establishing an experimental RD model, the expression of CYLD peaked on the third day and recovered to normal levels after 3 weeks, indicating that the high level of CYLD is an important contributor to cell death after RD. After RD surgery in rats with RNAi-mediated 

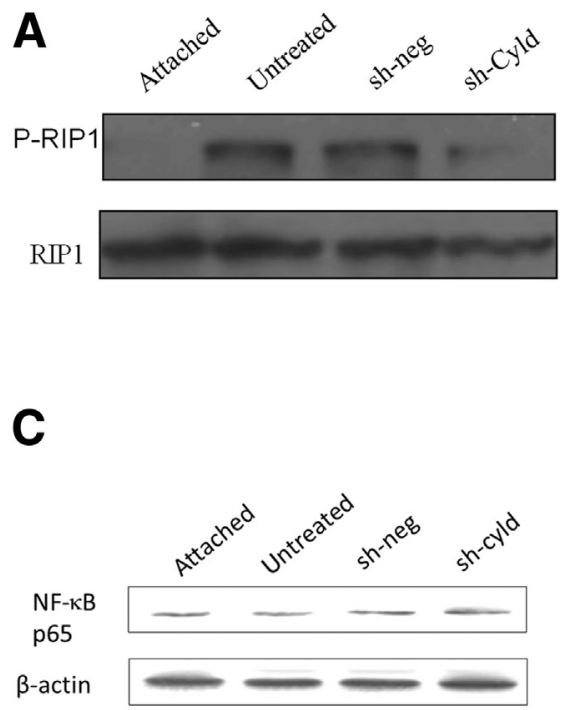

E

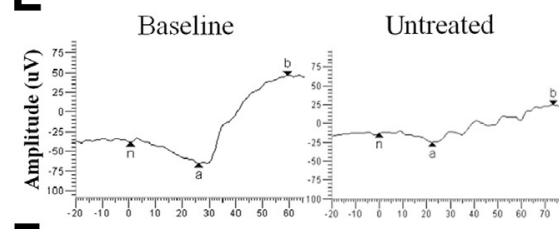

$\mathbf{F}$

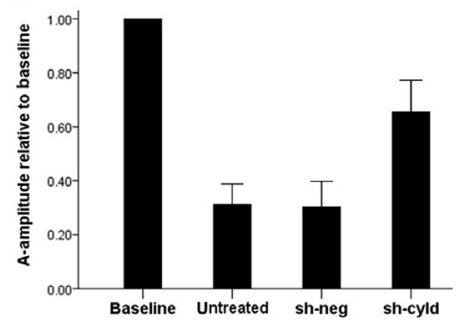

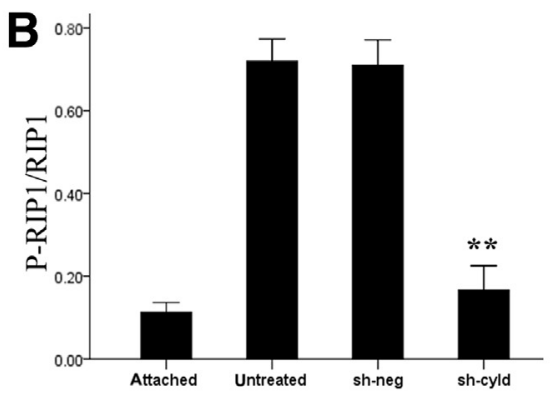
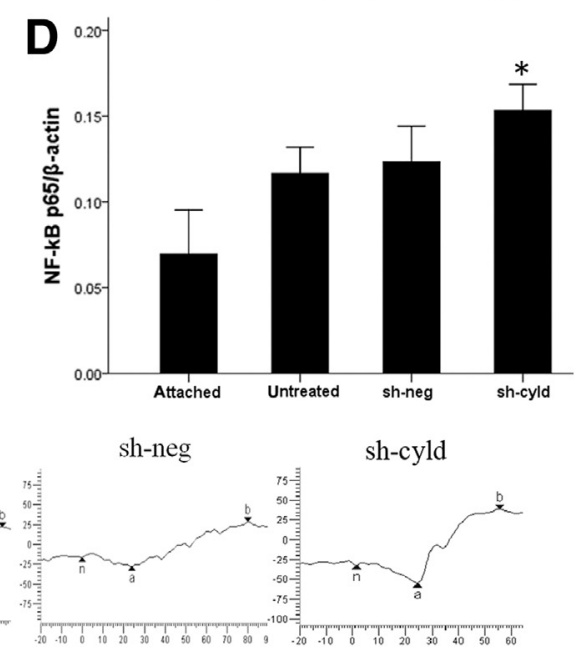

G

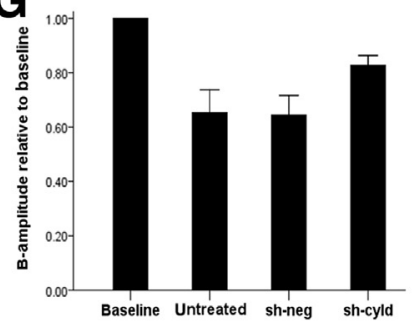

Figure 5 Receptor interacting protein-1 (RIP1) phosphorylation, NF- $\mathrm{BB}$ p65 expression, and electroretinography (ERG) a-wave after experimental retinal detachment in photoreceptor cells with decreased cylindromatosis (CYLD) expression. A and B: RIP1 phosphorylation in each group 3 days after experimental retinal detachment $(\mathbf{A})$ and the integrated optical density value (B). C and D: The protein expression level of NF- $\kappa B$ p65 with $\beta$-actin as a control. E: Change of ERG in each group. $n$ represents the resting potential at the beginning of ERG recording. $\mathbf{F}$ and $\mathbf{G}$ : Protective effect of decreased CYLD expression on ERG awave $(\mathbf{F})$ and b-wave $(\mathbf{G})$ against photoreceptor cell necroptosis. ${ }^{*} P<0.05,{ }^{*} P<0.01$ versus untreated and sh-neg group (analysis of variance). $a$, photoreceptors potential; b, bipolar cells potential; P-RIP1, phosphorylation-receptor interacting protein-1. knockdown of Cyld, the ubiquitination level of RIP1 increased in retinal cells, along with suppressed apoptosis and necroptosis of photoreceptor cells. This study further clarifies that the coregulatory mechanisms of CYLD in apoptosis and necroptosis of photoreceptor cells involve controlling the ubiquitination of RIP1. CYLD may be useful as an effective therapeutic option for RD-induced retinal damage.

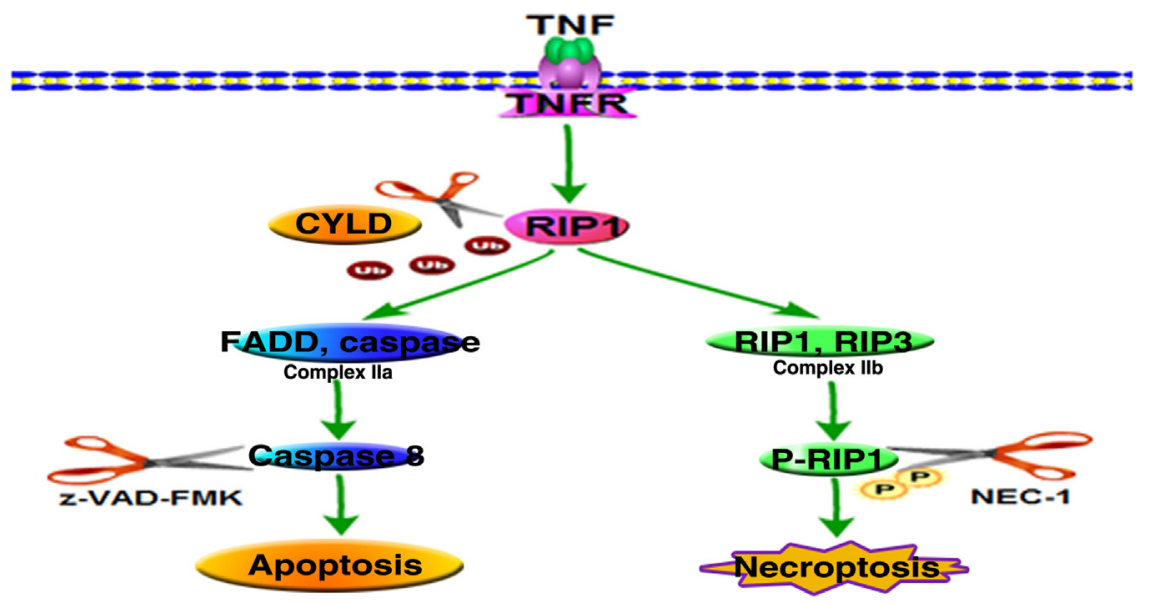

Figure 6 Ubiquitination and deubiquitination of receptor interacting protein-1 (RIP1) and cylindromatosis (CYLD) in the cell death signaling pathway. CYLD expression may increase after cell damage. Next, RIP1 might be deubiquitinated by CYLD and form complex IIa with FADD or complex IIb with RIP3 to function in apoptotic or necroptotic signaling and result in cell death. z-VAD-FMK can inhibit apoptosis by inhibiting the activation of caspase 8 , and Necrostatin- 1 can inhibit necroptosis via inhibiting the phosphorylation of RIP1. However, z-VAD-FMK and Necrostatin-1 cannot reduce cell death. CYLD can regulate apoptosis and necroptosis of photoreceptor cells by regulating the ubiquitination of RIP1. P, phosphorylation; P-RIP1, phosphorylation-receptor interacting protein-1; TNF, tumor necrosis factor; TNFR, tumor necrosis factor receptor; Ub, ubiquitination. 


\section{Acknowledgments}

We thank Yueqin Tang, Yue Liu, and Qing Gu, (Shanghai First People's Hospital, Shanghai Jiaotong University School of Medicine) for their assistance in this study.

\section{References}

1. Dong K, Sun X: Targeting death receptor induced apoptosis and necroptosis: a novel therapeutic strategy to prevent neuronal damage in retinal detachment. Med Hypotheses 2011, 77:144

2. Hisatomi T, Sakamoto T, Murata T, Yamanaka I, Oshima Y, Hata Y, Ishibashi T, Inomata H, Susin SA, Kroemer G: Relocalization of apoptosis-inducing factor in photoreceptor apoptosis induced by retinal detachment in vivo. Am J Pathol 2001, 158:1271-1278

3. Zacks DN, Hänninen V, Pantcheva M, Ezra E, Grosskreutz C, Miller JW: Caspase activation in an experimental model of retinal detachment. Invest Ophthalmol Vis Sci 2003, 44:1262-1267

4. Dong K, Zhu ZC, Wang FH, Ke GJ, Yu Z, Xu X: Activation of autophagy in photoreceptor necroptosis after experimental retinal detachment. Int J Ophthalmol 2014, 7:745-752

5. Dong K, Zhu H, Song Z, Gong Y, Wang F, Wang W, Zheng Z, Yu Z, Gu Q, Xu X, Sun X: Necrostatin-1 protects photoreceptors from cell death and improves functional outcome after experimental retinal detachment. Am J Pathol 2012, 181:1634-1641

6. Trichonas G, Donahoe PK: Receptor interacting protein kinases mediate retinal detachment-induced photoreceptor necrosis and compensate for inhibition of apoptosis. Proc Natl Acad Sci U S A 2010, 107:21695-21700

7. Degterev A, Huang Z, Boyce M, Li Y, Jagtap P, Mizushima N, Cuny GD, Mitchison TJ, Moskowitz MA, Yuan J: Chemical inhibitor of nonapoptotic cell death with therapeutic potential for ischemic brain injury. Nat Chem Biol 2005, 1:112-119

8. Hitomi J, Christofferson DE, Ng A, Yao J, Degterev A, Xavier RJ, Yuan J: Identification of a molecular signaling network that regulates a cellular necrotic cell death pathway. Cell 2008, 135:1311-1323

9. Degterev A, Hitomi J, Germscheid M, Ch'en IL, Korkina O, Teng X, Abbott D, Cuny GD, Yuan C, Wagner G, Hedrick SM, Gerber SA, Lugovskoy A, Yuan J: Identification of RIP1 kinase as a specific cellular target of necrostatins. Nat Chem Biol 2008, 4:313-321

10. Wright A, Reiley WW, Chang M, Jin W, Lee AJ, Zhang M, Sun SC: Regulation of early wave of germ cell apoptosis and spermatogenesis by deubiquitinating enzyme CYLD. Dev Cell 2007, 13:705-716

11. Wang L, Du F, Wang X: TNF-alpha induces two distinct caspase-8 activation pathways. Cell 2008, 133:693-703

12. Trompouki E, Hatzivassiliou E, Tsichritzis T, Farmer H, Ashworth A, Mosialos G: CYLD is a deubiquitinating enzyme that negatively regulates NF-kappaB activation by TNFR family members. Nature 2003, 424:793-796

13. Wertz IE, Dixit VM: Regulation of death receptor signaling by the ubiquitin system. Cell Death Differ 2010, 17:14-24

14. Zhu H, Qian J, Wang W, Yan Q, Xu Y, Jiang Y, Zhang L, Lu F, Hu W, Zhang X: RNA interference of GADD153 protects photoreceptors from endoplasmic reticulum stress-mediated apoptosis after retinal detachment. PLoS One 2013, 8:e59339

15. Greenberg KP, Lee ES, Schaffer DV, Flannery JG: Gene delivery to the retina using lentiviral vectors. Adv Exp Med Biol 2006, 572: 255-266

16. Bennett J, Chung DC, Maguire A: Gene delivery to the retina: from mouse to man. Methods Enzymol 2012, 507:255-274

17. Wu WC, Lai CC, Chen SL, Xiao X, Chen TL, Tsai RJ, Kuo SW, Tsao YP: Gene therapy for detached retina by adeno-associated virus vector expressing glial cell line-derived neurotrophic factor. Invest Ophthalmol Vis Sci 2002, 43:3480-3488
18. Takahashi M: Delivery of genes to the eye using lentiviral vectors Methods Mol Biol 2004, 246:439-449

19. Kessel L, Tendal B, Jørgensen KJ, Erngaard D, Flesner P, Andresen JL, Hjortdal J: Post-cataract prevention of inflammation and macular edema by steroid and nonsteroidal anti-inflammatory eye drops: a systematic review. Ophthalmology 2014, 121:1915-1924

20. Cui Y-L, Wang L, Tian Z-T, Lin Z-F, Chen D-C: The effect of rhubarb pre-treatment on intestinal microcirculation in septic rats. Am J Chin Med 2014, 42:1215-1227

21. Linberg KA, Lewis GP, Matsumoto B, Fisher SK: Immunocytochemical evidence that rod-connected horizontal cell axon terminals remodel in response to experimental retinal detachment in the cat. Mol Vis 2006, 12:1674-1686

22. Sun X, Xu X, Wang F, Zhang X, Ho PC, Liu H, Qian J, Yu Z, Lu H, $\mathrm{Xu} \mathrm{W}$ : Nerve growth factor helps protect retina in experimental retinal detachment. Ophthalmologica 2008, 222:58-61

23. Kroemer G, Galluzzi L, Vandenabeele P, Abrams J, Alnemri ES, Baehrecke EH, Blagosklonny MV, El-Deiry WS, Golstein P, Green DR, Hengartner M, Knight RA, Kumar S, Lipton SA, Malorni W, Nunez G, Peter ME, Tschopp J, Yuan J, Piacentini M, Zhivotovsky B, Melino G: Classification of cell death: recommendations of the Nomenclature Committee on Cell Death 2009. Cell Death Differ 2009, 16:3-11

24. You Z, Savitz SI, Yang J, Degterev A, Yuan J, Cuny GD, Moskowitz MA, Whalen MJ: Necrostatin-1 reduces histopathology and improves functional outcome after controlled cortical impact in mice. J Cereb Blood Flow Metab 2008, 28:1564-1573

25. Xu X, Chua CC, Kong J, Kostrzewa RM, Kumaraguru U, Hamdy RC, Chua BH: Necrostatin-1 protects against glutamate-induced glutathione depletion and caspase-independent cell death in HT-22 cells. J Neurochem 2007, 103:2004-2014

26. Cao W, Tombran-Tink J, Elias R, Sezate S, Mrazek D, McGinnis JF: In vivo protection of photoreceptors from light damage by pigment epithelium-derived factor. Invest Ophthalmol Vis Sci 2001, 42: $1646-1652$

27. Wu P, Shi K, An J, Ci Y, Li F, Hui K, Yang Y, Xu C: The LEF1/CYLD axis and cIAPs regulate RIP1 deubiquitination and trigger apoptosis in selenite-treated colorectal cancer cells. Cell Death Dis 2013, 5:e1085

28. Gandhi AK, Kang J, Havens CG, Conklin T, Ning Y, Wu L, Ito T, Ando H, Waldman MF, Thakurta A: Immunomodulatory agents lenalidomide and pomalidomide co-stimulate $\mathrm{T}$ cells by inducing degradation of $\mathrm{T}$ cell repressors Ikaros and Aiolos via modulation of the E3 ubiquitin ligase complex CRL4CRBN. Br J Haematol 2014, 164:811-821

29. Zhou H-R, Jia Q, Pestka JJ: Ribotoxic stress response to the trichothecene deoxynivalenol in the macrophage involves the SRC family kinase Hck. Toxicol Sci 2005, 85:916-926

30. Declercq W, Vanden Berghe T, Vandenabeele P: RIP kinases at the crossroads of cell death and survival. Cell 2009, 138:229-232

31. Bignell GR, Warren W, Seal S, Takahashi M, Rapley E, Barfoot R, Green H, Brown C, Biggs PJ, Lakhani SR, Jones C, Hansen J, Blair E, Hofmann B, Siebert R, Turner G, Evans DG, Schrander-Stumpel C, Beemer FA, van Den Ouweland A, Halley D, Delpech B, Cleveland MG, Leigh I, Leisti J, Rasmussen S: Identification of the familial cylindromatosis tumour-suppressor gene. Nat Genet 2000, 25:160-165

32. Martinon F, Burns K, Tschopp J: The inflammasome: a molecular platform triggering activation of inflammatory caspases and processing of proIL-beta. Mol Cell 2002, 10:417-426

33. Tinel A, Tschopp J: The PIDDosome, a protein complex implicated in activation of caspase-2 in response to genotoxic stress. Science 2004, 304:843-846

34. Christofferson DE, Yuan J: Necroptosis as an alternative form of programmed cell death. Curr Opin Cell Biol 2010, 22:263-268

35. Cho YS, Challa S, Moquin D, Genga R, Ray TD, Guildford M, Chan FK: Phosphorylation-driven assembly of the RIP1-RIP3 complex regulates programmed necrosis and virus-induced inflammation. Cell 2009, 137:1112-1123 